\title{
Diabetes and periodontitis
}

\author{
Robert Hirsch, Senior Lecturer, Dental School, The University of Adelaide, Adelaide
}

\section{Summary}

Chronic gingivitis and periodontitis are common inflammatory conditions of the periodontal tissues. Given the 'right' concurrence of risk factors, a person with periodontitis can experience significant destruction of tooth-supporting bone, ultimately resulting in tooth loss. Poorly controlled diabetes is an important risk factor for periodontitis, and gingivitis and periodontitis are sometimes the first sign that a patient has diabetes. As severe periodontitis can lead to the loss of teeth, it is important that patients with diabetes practise good oral hygiene and have regular dental check-ups so that problems can be detected quickly.

Key words: gingivitis, dental implications.

(Aust Prescr 2004;27:36-8)

\section{Introduction}

In chronic periodontitis, the tooth supporting structures (alveolar bone and the periodontal ligament) are destroyed. The disease has a multifactorial origin. Complexes of commensal oral anaerobic bacteria and perhaps viruses are thought to interact with risk factors, such as smoking, diabetes and depression, to create the conditions which make a person susceptible to periodontitis. The patient's immuno-inflammatory response to the bacteria causes the tissue destruction which occurs in chronic periodontitis. Less than $5 \%$ of Australians are susceptible to the severe periodontitis which results in tooth loss, although up to $10 \%$ experience moderate amounts of periodontitis-related bone loss.

It is useful to distinguish chronic gingivitis from periodontitis. Chronic gingivitis is the very common inflammatory reaction occurring in the gingival tissues in response to the accumulation of dental plaque. It usually precedes the development of periodontitis, but chronic gingivitis does not inevitably progress to periodontitis. The clinical appearance of gingivitis may be modified by systemic factors such as poorly controlled diabetes, which can significantly accentuate the gingival tissues' response to dental plaque (Fig. 1).

\section{The relationship between diabetes, gingivitis and periodontitis}

Although periodontitis is a recognised complication of diabetes, people with well-controlled diabetes who have good oral hygiene are not at increased risk of periodontitis. However, their susceptibility to periodontitis is significantly increased when their diabetes is poorly controlled, particularly if they also smoke.

Recent epidemiologic evidence shows that the prevalence of diabetes in patients with periodontitis is significantly greater (by two times) than in people without periodontitis. ${ }^{1}$ Given that diabetes may be present for a number of years before it is diagnosed, and that the prevalence of diabetes is increasing in the Australian community, dentists may be the first health professionals to detect a patient's diabetes. The gingival and periodontal signs which may alert the clinician that the patient has previously undiagnosed diabetes or that the patient's diabetes is poorly controlled, include:

- persistence of gingival inflammation after standard periodontal treatment (thorough supra- and subgingival scaling and cleaning, oral hygiene instruction)

- severe gingival inflammatory response to plaque and proliferation of gingival tissues at the gingival margin (Fig. 1)

Fig. 1

Severe gingival inflammatory reaction to dental plaque This 55-year-old patient has recently diagnosed type 2 diabetes. The gingival tissue is friable, oedematous and prone to bleeding on tooth brushing.

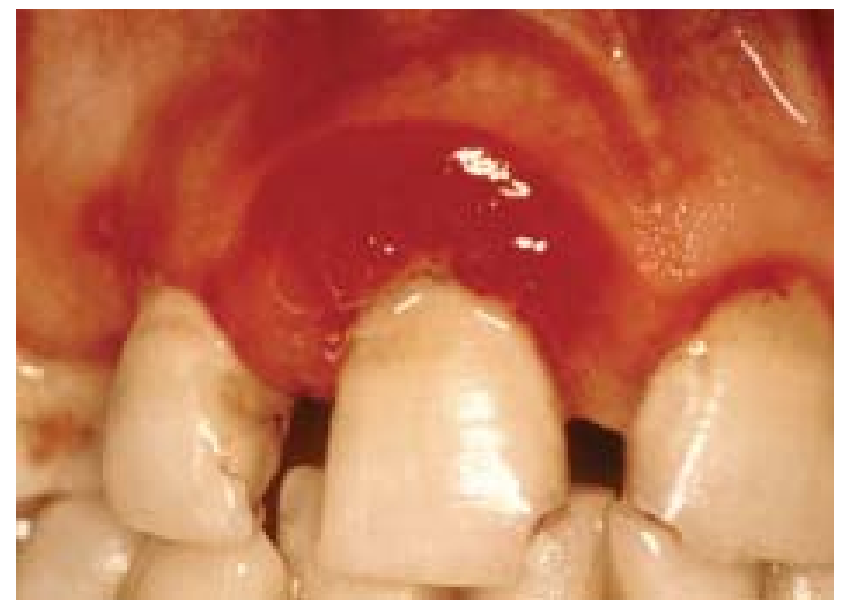


continuing alveolar bone loss despite periodontal treatment (Fig. 2)

- severe, aggressive periodontitis in people $20-45$ years of age (deep periodontal pocketing, increased tooth mobility and tooth migration, causing teeth to over-erupt or spaces to open between teeth, and radiographic evidence of advanced bone loss)

- simultaneous formation of multiple periodontal abscesses (Fig. 3). ${ }^{2}$

\section{Fig. 2}

\section{Alveolar bone loss}

This orthopantomograph of a 54-year-old patient with poorly controlled diabetes shows extensive alveolar bone loss involving most teeth. The destruction of bone has been rapid even though the patient has been undergoing periodontal therapy.

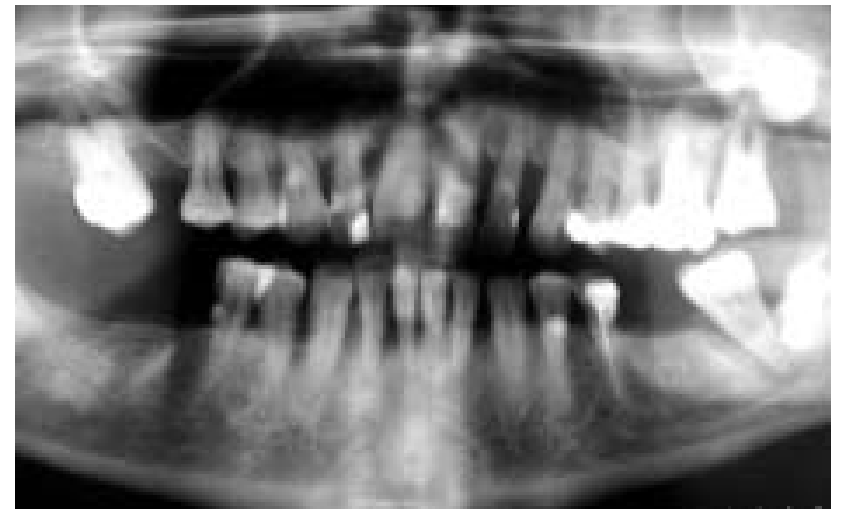

\section{Fig. 3}

\section{Periodontal abscess formation}

This 56-year-old patient has poorly controlled type 2 diabetes. The lower left lateral incisor $(*)$ is very mobile and has migrated from its usual location in the arch. The tooth has lost more than two-thirds of its supporting bone because of periodontitis.

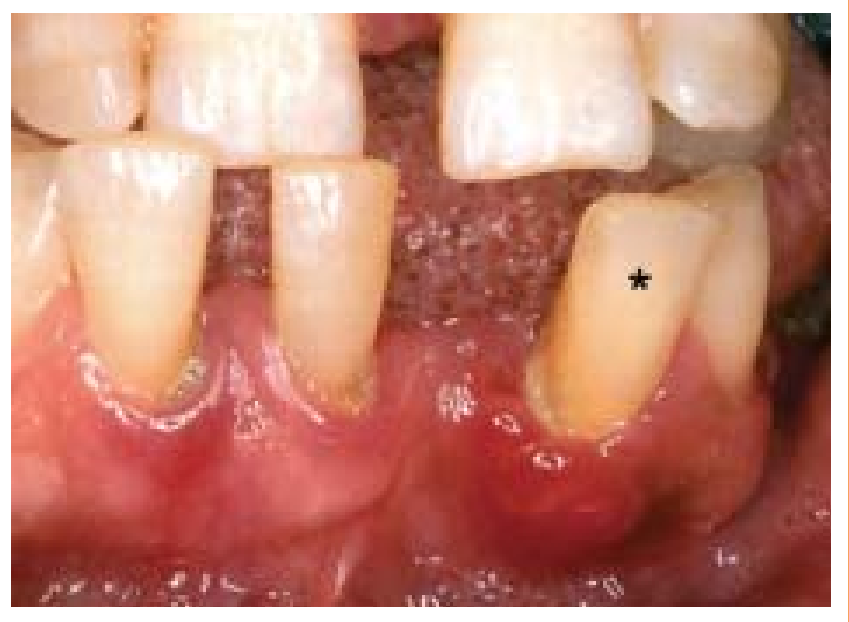

\section{How diabetes increases susceptibility to periodontitis}

Advanced glycation end products deposited in the tissues as a result of hyperglycaemia can alter the phenotype of macrophages and other cells via a specific cell-surface receptor. Macrophages are key cells in the pathogenesis of periodontitis through their ability to produce a large array of cytokines. They also influence the inflammatory response, the metabolism of fibroblasts and lymphocytes and stimulate bone resorption via prostaglandin $E_{2}$. It is thought that the advanced glycation end products transform the macrophages into cells with a destructive phenotype, producing pro-inflammatory cytokines in an uncontrolled fashion. ${ }^{3}$

Neutrophils are the primary defence cells of the periodontium. The reduced neutrophil function observed in patients with diabetes is therefore another mechanism increasing the susceptibility to periodontitis.

\section{Is there a relationship between periodontitis and the ability to control diabetes?}

While periodontitis is a recognised complication of poorly controlled diabetes, it has been proposed that severe periodontitis may make the metabolic control of diabetes more difficult. The process may be mediated through the systemic release of inflammatory cytokines (e.g. TNF- $\alpha$ ) from periodontitis lesions, and chronic, low-level systemic exposure to Gram negative organisms. Although early studies have been poorly designed, one of these suggested that when antibiotics were added to standard periodontal treatment (debridement of the teeth and oral hygiene improvement), diabetic control improved significantly for a period of three months. ${ }^{2}$

\section{What can the patient do?}

People with diabetes need to practise high standards of daily oral hygiene, including brushing and flossing. The use of interdental brushes (which are like small bottle brushes) is indicated where there has been some recession of the gingivae. Adjunctive use of a chlorhexidine mouthwash $(0.12 \%)$ or chlorhexidine gel $(0.2 \%)$ twice daily (used independently of toothpaste so that the chlorhexidine is not inactivated) may be useful in controlling the more severe forms of gingivitis. Patients should consult with their dentist or periodontist regarding the recommended duration of use of chlorhexidine. Dental care, which is specifically aimed at monitoring the health of the periodontal tissues and providing the necessary treatment, is needed at six-month intervals.

Medical practitioners who suspect a patient has diabetes-related gingivitis or periodontitis should ensure that an early referral is made to a dentist. Dentists in turn need to refer advanced or suspect cases to a periodontist. 


\section{Conclusion}

Medical and dental practitioners need to be aware of the interrelationship between poorly controlled or undiagnosed diabetes mellitus and chronic gingivitis and periodontitis. This is particularly important because of the rising prevalence of diabetes in the Australian community.

\section{References}

1. Soskolne WA, Klinger A. The relationship between periodontal diseases and diabetes: an overview. Ann Periodontol 2001;6:91-8.

2. Bjelland S, Bray P, Gupta N, Hirscht R. Dentists, diabetes and periodontitis. Aust Dent J 2002;47:202-7.
3. Mealy B. Position paper. Diabetes and periodontal diseases. J Periodontol 2000;71:664-78.

http://www.perio.org:80/resources-products/pdf/4-diabetes. pdf [cited 2004 March]

Conflict of interest: none declared

Photographs courtesy of the author

\section{Self-test questions}

The following statements are either true or false (answers on page 51)

3. Periodontitis may be the first presentation of diabetes.

4. Gingivitis precedes periodontitis.

\section{Introducing the Adverse Medicine Events Line 1300134237}

Traditionally, only health professionals have reported adverse drug reactions to the Adverse Drug Reactions Advisory Committee (ADRAC). However, recent studies have shown that when consumers report adverse drug reactions, the reports are received more quickly, about a broader range of reactions and give a better account of the event. In addition, adverse reactions from complementary medicines are more likely to be reported by consumers.

Consumer reporting of adverse drug reactions has become a reality in Australia with the launch of a new telephone hotline called the Adverse Medicine Events (AME) Line. Operated by clinical pharmacists from Mater Health Services in Brisbane, the AME Line is a telephone service through which consumers can seek information about or report adverse events associated with medicines, including adverse drug reactions, errors and so-called 'near misses'.

The service, which is an initiative of the Australian Council for Safety and Quality in Health Care was launched in October 2003. It will operate from 9 am to $6 \mathrm{pm}$, Monday to Friday for an initial period of 18 months. Objectives of the AME Line are to:

- create a system for consumers to report adverse experiences with medicines
- promote openness and accuracy regarding adverse medicine events

- identify trends in adverse medicine events to know when, where and how things go wrong

- ultimately integrate the information into health systems, to improve safety and quality.

The pharmacists operating the AME Line can provide information or answer questions regarding medication-related adverse effects and ensure that adverse drug reaction reports satisfying specific criteria are submitted to ADRAC. Individuals are not named in these reports, but rather safety and quality issues within the system are identified. Medication errors and 'near misses' are reported to the Australian Council for Safety and Quality in Health Care. De-identified data will be collected and fed back to health professionals to assist in changing systems to help prevent the recurrence of these adverse events. Health professionals are encouraged to refer consumers to the AME Line, for information, advice and reporting of adverse medicine events. Further information about the line is available at: www.safetyandquality.org.au or by contacting Geraldine Moses orTreasure McGuire on (07) 38408087. 